\title{
The Lateral Plantar Artery as Recipient Vessel for Microsurgical Lateral Plantar Forefoot Reconstruction: Case Report
}

\author{
Yen-Chou Chen, $\mathrm{MD}^{1} *$; Mario F. Scaglioni, $\mathrm{MD}^{2}$ \\ ${ }^{1}$ Department of Plastic and Reconstructive Surgery, Body Science \& Metabolic Disorders International (BMI) Medical Center, \\ China Medical University Hospital, Taichung, Taiwan \\ ${ }^{2}$ Department of Plastic and Hand Surgery, University Hospital Zurich, Zurich, Switzerland
}

\begin{abstract}
Reconstruction of the lateral plantar forefoot defect remains a challenge. With the advent of microsurgical techniques, free flaps have gained popularity in plantar forefoot reconstruction. However, the choices of recipient vessels are limited in this area. Therefore, we report two cases with lateral plantar forefoot defects using the lateral plantar artery as recipient vessel for free flap reconstruction. Flaps (me dial plantar flap and peroneal artery perforator flap) survived completely. The postoperative course was uneventful and the patients were able to resume normal ambulation. The dissection of lateral plantar artery was simple and straightforward. Its vascular caliber was adequate for microvascular anastomosis. These advantages might make it a valuable option as the recipient vessel choice for lateral plantar forefoot reconstruction.
\end{abstract}

\section{Introduction}

Reconstruction of the plantar forefoot area remains a challenging problem due to the limited alternatives. With the advent of microsurgical techniques, free flaps become more and more popularly employed in plantar forefoot reconstruction [1-7]. Although the choice of an optimal flap is very important, the selection of appropriate recipient vessels is also equally crucial. Failure to identify appropriate recipient vessels may result in size discrepancy, unnecessary use of vein graft, and eventually flap loss. The lateral plantar artery, one of the dominant vessels contributing plantar foot circulation, is rarely reported to be used as recipient vessel for free flap reconstruction. Here, we report two cases with lateral plantar forefoot defects where the lateral plantar artery was used as recipient vessel for free flap reconstruction. The case reports describe the relevant surgical anatomy and discuss the role in the plantar forefoot reconstruction.

\section{Anatomy and landmark of lateral plantar artery}

The lateral plantar artery originating from the posterior tibial artery runs in the posterior compartment of the tarsal tunnel and continues obliquely under the tarsus in the direction to the base of the fifth metatarsal bone.

\section{*Correspondence: Yen-Chou Chen, MD}

Department of Plastic and Reconstructive Surgery, Body Science \& Metabolic Disorders International Medical Center, China Medical University Hospital, Taichung, Taiwan. E-mail: chenrenslo@gmail.com

Dr. Chen and Dr. Scaglioni contributed to this paper equally as co-first author.
It travels downward obliquely between the flexor digitorum brevis muscle and the quadratus plantae after bifurcation with medial plantar artery in the second plantar layer. It then proceeds superficially between the flexor digitorum brevis and the abductor digiti minimi muscle under the plantar aponeurosis. At approximately $1.2 \mathrm{~cm}$ distal to the tuberosity of the fifth metatarsal bone, the lateral plantar artery divides into superficial (the little toe fibular artery) and deep (deep plantar arch) branches [8]. To locate the direction of the lateral plantar artery, a reference line is drawn from

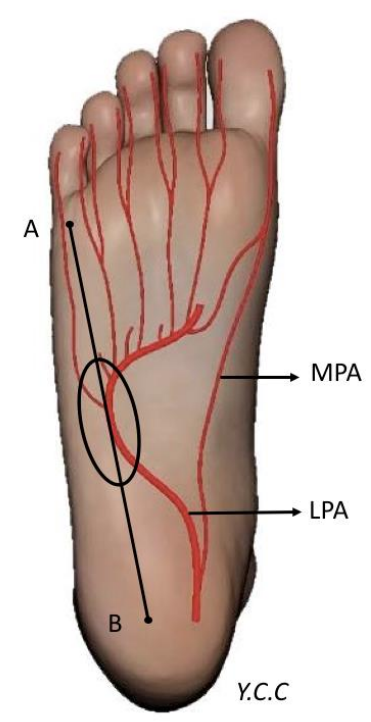

Figure 1. A reference line is drawn from point A (base of fifth toe) to point $B$ (heel). The middle third of the reference line (circle) represents the portion of lateral plantar artery that could be used as recipient vessel. MPA, medial plantar artery; LPA, lateral plantar artery. 


\section{Case Report}

the base of the fifth toe base to the heel. The middle third of the line indicates the part of the lateral plantar artery that potentially can be used as recipient vessel (Figure 1).

\section{Case 1}

A 60-year-old female patient was presented due to malignant melanoma measuring $2.0 \mathrm{~cm} \times 1.2 \mathrm{~cm}$ on her right lateral plantar forefoot. Wide excision with sentinel lymph node dissection was scheduled (Figure 2A). Wide excision of the tumor with $2 \mathrm{~cm}$ safety margin was done with clear margin proven by frozen section. A $5 \mathrm{~cm} \times 5 \mathrm{~cm}$ full-thickness defect was created and medial plantar flap was designed according to the defect size on the same foot. The flap was elevated in conventional manner (Figure 2B). The location of lateral plantar artery was confirmed cial branch of lateral plantar artery was dissected out along with one concomitant vein. The diameters of the artery and the vein were $1.8 \mathrm{~mm}$ and $2.2 \mathrm{~mm}$ respectively. The fifth toe circulation was tested before ligation of the artery. The medial plantar flap was then divided and transferred to reconstruct the defect. The medial plantar artery was anastomosed to the superficial branch of lateral plantar artery (Figure 2C). The deep branch of lateral plantar artery was preserved. Venous outflow was ensured by repairing one concomitant vein. The instep donor site was covered with thin fullthickness skin graft. The viability of flap was good without complications in the postoperative course. The patient was able to achieve full-weight bearing on right foot, 2 months after the surgery. The contour of the flap was excellent (Figure 2D).

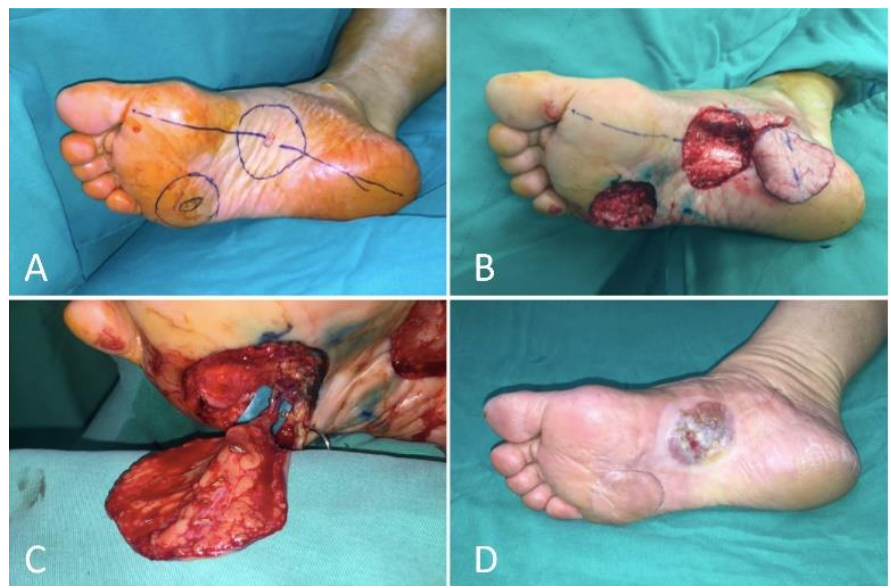

Figure 2. (A) Malignant melanoma over right lateral plantar forefoot and design of the ipsilateral medial plantar flap. (B) After wide excision, a $5 \times 5 \mathrm{~cm}$ soft tissue defect was left. The medial plantar flap was elevated. (C) The pedicle of medial plantar flap was anastomosed to the superficial branch of lateral plantar artery and one concomitant vein. (D) The flap healed well with satisfactory contour in 3 months after the surgery.

\section{Case 2}

A 57-year-old female patient suffered from crushing injury on the right foot during a traffic accident and resulted in a full-thickness soft tissue defect measuring $18.0 \mathrm{~cm}$ in length and $5.0 \mathrm{~cm}$ in width (Figure $3 \mathrm{~A}$ ). An 18 $\mathrm{cm} \times 6 \mathrm{~cm}$ peroneal artery perforator (PAP) flap was designed on the ipsilateral lateral leg (Figure 3B). The flap dissection technique was the same as described in our previous publication [4]. The location of lateral plantar artery was checked by hand-held Doppler probe. After dividing the plantar fascia and separating the junction between the flexor digitorum brevis muscle and the abductor digiti minimi, the lateral plantar artery along with one accompanying vein was dissected out. The diameters of the artery and the vein were $2.2 \mathrm{~mm}$ and $3.0 \mathrm{~mm}$ respectively. The artery was temporarily clamped to ensure the distal foot circulation. The flap was then transferred and microvascular anastomosis was performed (Figure

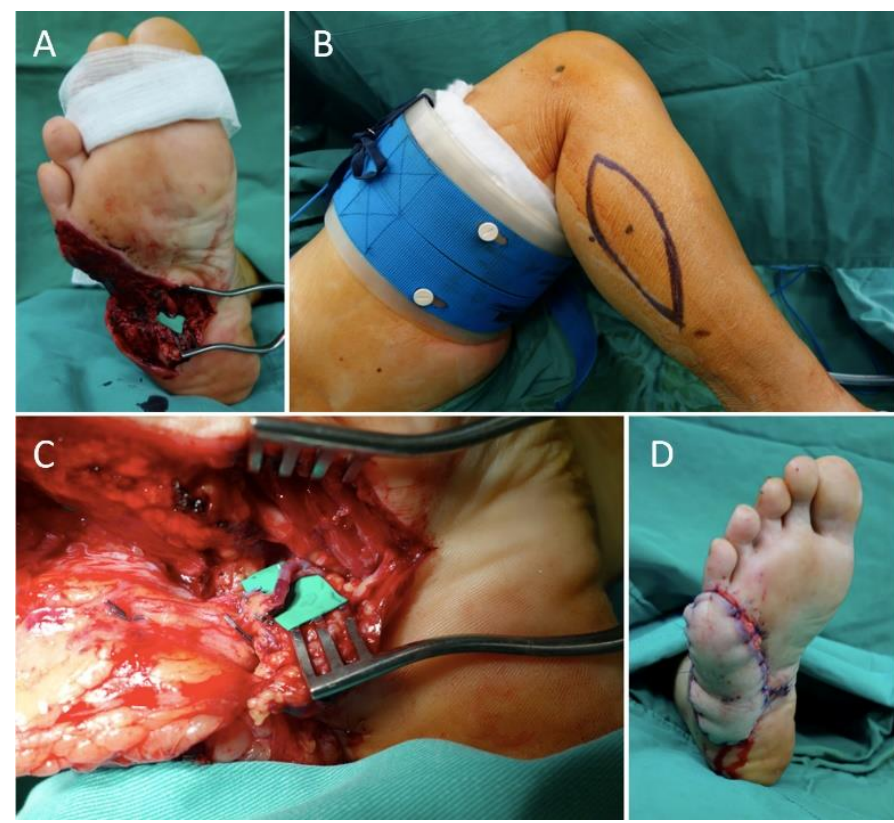

Figure 3. (A) A traumatic soft tissue defect from lateral plantar forefoot extending to middle plantar foot. The lateral plantar artery was dissected and prepared as recipient vessel. (B) A proximal peroneal artery perforator flap was designed on the ipsilateral lateral leg. (C) The flap was transferred to reconstruct the defect. Microvascular anastomosis was performed to the lateral plantar artery and one concomitant vein. (D) The immediate appearance after flap reconstruction.

3C). The flap perfusion was satisfactory at the time of finishing the anastomosis (Figure 3D). The postoperative course was uneventful. Partial weight bearing was allowed one month after the surgery. Patient was able to achieve full weight bearing and normal ambulation 3 months later without difficulty in wearing the shoe.

\section{Discussion}

The ideal coverage to plantar forefoot is to use durable soft tissue with minimal bulkiness and follow the principle of "replace like tissue with like" [1]. Therefore, glabrous plantar skin from the spare part of foot is the first choice. Several local flap options have been described to reconstruct the plantar forefoot defect, including V-Y plantar flap [9], plantar marginal septum cutaneous island flap [10], island lateral plantar artery perforator flap [11], and distally based or reverse medial plantar flap $[3,12,13]$. In particular, the conventional flap design of the medial plantar flap has often limited mobilization, but if based on its retrograde flow, the arch of movement of the flap can greatly improve. However, venous congestion is a concern, requiring supercharging or delay procedure $[14,15]$. The local flap option is summarized in table 1 . When the defect is large $(>100$ $\mathrm{cm} 2$ ) or the foot is severely traumatized, a reconstruction with free flaps is indicated [16]. Although the best flap type has not yet been determined, the instep flap, due to its similar viscoelasticity to the plantar forefoot skin, is undoubtedly the first choice $[2,5,16,17]$ Thin perforator flap $(<6 \mathrm{~mm}$ in thickness), composed of small fat lobules invested by dense fibrous septa allowing less gliding, is another optimal choice $[4,7]$. Furthermore, sensate coverage is available by including branch of medial plantar nerve with the medial plantar flap, which is crucial for plantar defect reconstruction. In the case 1, although the defect was a candidate for a reverse medial plantar flap, we were concerned about the uncertainty of the venous drainage and felt safer to perform free flap reconstruction. In the case 2 , the defect was $18 \mathrm{~cm}$ in length, which exceeded the conventional design of medial plantar flap [5]. The peroneal artery perforator (PAP) flap provided a thin and pliable skin [4]. Based on our previous experience, using it in the distal hand and foot reconstruction, we successfully transferred 
Table 1. Local flap option for plantar foot reconstruction

\begin{tabular}{lll} 
Flap Type & Advantage & Disadvantage \\
\hline V-Y plantar flap & $\begin{array}{l}\text { Easy design and } \\
\text { dissection }\end{array}$ & Limited movement \\
\hline $\begin{array}{l}\text { Plantar marginal } \\
\text { septum cutaneous } \\
\text { island flap }\end{array}$ & $\begin{array}{l}\text { For medial plantar } \\
\text { foot reconstruction }\end{array}$ & Meticulous dissection \\
\hline $\begin{array}{l}\text { Island lateral plantar } \\
\text { artery perforator flap }\end{array}$ & $\begin{array}{l}\text { For lateral plantar } \\
\text { foot reconstruction }\end{array}$ & Meticulous dissection \\
\hline $\begin{array}{l}\text { Reverser medial plantar } \\
\text { flap }\end{array}$ & $\begin{array}{l}\text { Versatile flap design } \\
\text { Large arc of rotation } \\
\text { and flap movement }\end{array}$ & $\begin{array}{l}\text { Meticulous dissection } \\
\text { May need venous } \\
\text { supercharge or delay } \\
\text { procedure }\end{array}$ \\
\hline
\end{tabular}

the PAP flap for resurfacing of the plantar forefoot defect.

Other than the flap type, the recipient vessels are of paramount importance. The dorsal metatarsal artery, dorsalis pedis artery, medial plantar artery, and posterior tibial artery have been well reported as recipient vessels in the literature $[1-3,6,18]$. Particularly, the dorsal metatarsal artery and the dorsalis pedis artery are suitable recipient vessels for dorsal foot and transmetatarsal foot defects; but not for pure plantar forefoot defect. The use of the medial plantar artery or posterior tibial artery is more convenient in this situation. Zelken et al. reported the use of innervated medial plantar flaps for forefoot reconstruction and mentioned that if ipsilateral medial plantar flap was employed, it required the interposition of a vein graft in order to reach the ipsilateral medial plantar artery or posterior tibial artery [18]. Size discrepancy was encountered between donor vein graft and recipient artery, which increased the chance of thrombosis. For defects mainly located in the lateral part of plantar forefoot, as in our two cases, the recipient vessels, the just above mentioned, are too far away to reach. Although it is possible to use the plantar

Table 2. Recipient vessel choice in plantar forefoot reconstruction

\begin{tabular}{|lll|}
\hline Recipient Vessel & Advantage & Disadvantage \\
\hline Dorsalis pedis artery & $\begin{array}{l}\text { Easy dissection } \\
\text { and exposure }\end{array}$ & Need pass through tunnel \\
\hline Dorsal metatarsal artery & $\begin{array}{l}\text { Easy dissection } \\
\text { and exposure }\end{array}$ & Need pass through tunnel \\
\hline Posterior tibial artery & $\begin{array}{l}\text { Easy dissection } \\
\text { and exposure }\end{array}$ & Need vein graft \\
\hline Medial plantar artery & Nearby the defect & May have size discrepancy \\
\hline
\end{tabular}

metatarsal artery, the diameter is usually small and requires super-microsurgical technique; moreover, the presence of accompanying vein is unpredictable [19]. The recipient vessel choice for plantar forefoot reconstruction is summarized in table 2 . The lateral plantar artery is rarely reported as recipient vessel due to its deep location. However, in the middle third of the reference line we have proposed, the lateral plantar artery runs superficially between the flexor digitorum brevis and abductor digiti minimi [8]. It can be easily exposed by incision through the plantar aponeurosis and separation of the muscles, the vascular diameter is closed to the flap pedicle size.

\section{Conclusion}

Division of the lateral plantar artery does not jeopardize the foot circulation because of anastomosis of the lateral plantar artery with the dorsalis pedis artery at the first intermetatarsal space. However, care should be taken with patients with peripheral artery occlusive disease and the flow of dorsalis pedis artery should be confirmed before surgery. Given the advantages of sizable vessel, easy dissection, and proximity to the defect, we believe that the lateral plantar artery might be a valuable option as recipient vessel for lateral plantar forefoot reconstruction.

\section{Keywords}

Plantar forefoot reconstruction; peroneal artery perforator flap; medial plantar flap; lateral plantar artery.

\section{Abbreviations}

PAP, peroneal artery perforator; MPA, medial plantar artery; LPA, lateral plantar artery.

\section{Article Information \\ Conflict of Interest Disclosures: None Funding: None}

\section{References}

1. Hollenbeck ST, Woo S, Komatsu I, Erdmann D, Zenn MR, Levin LS. Longitudinal outcomes and application of the subunit principle to 165 foot and ankle free tissue transfers. Plastic and Reconstructive Surgery 2010; 125: 924-934

2. Lykoudis EG, Seretis K, Lykissas MG. Free sensate medial plantar flap for contralateral plantar forefoot reconstruction with flap reinnervation using end-to-side neurorrhaphy: a case report and literature review. Microsurgery 2013; 33: 227-231.

3. Oh SJ, Moon M, Cha J, Koh SH, Chung $\mathrm{CH}$. Weight-bearing plantar reconstruction using versatile medial plantar sensate flap. Journal of Plastic, Reconstructive \& Aesthetic Surgery: JPRAS 2011; 64: 248-254.

4. Scaglioni MF, Kuo YR, Chen YC. Reconstruction of distal hand and foot defects with the free proximal peroneal artery perforator flap. Microsurgery 2016; 36:183-190.

5. Scheufler O, Kalbermatten D, Pierer G. Instep free flap for plantar soft tissue reconstruction: indications and options. Microsurgery 2007; 27: 174-180

6. Zhu YL, Wang $\mathrm{Y}$, He XQ, Zhu M, Li FB, Xu YQ. Foot and ankle reconstruction: an experience on the use of 14 different flaps in 226 cases. Microsurgery 2013; 33: 600-604.

7. Hong JP, Kim EK. Sole reconstruction using anterolateral thigh perforator free flaps. Plastic and Reconstructive Surgery 2007; 119: 186193.

8. Li L, Song $\mathrm{D}$, Zheng $\mathrm{H}$, et al. Anatomical basis of the reverse lateral plantar artery perforator flap design. Surgical and Radiologic Anatomy 2015; 37: 983-988.

9. Colen LB, Replogle SL, Mathes SJ. The V-Y plantar flap for reconstruction of the forefoot. Plastic and Reconstructive Surgery 1988; 81: 220-228.

10. Bertelli JA, Duarte HE. The plantar marginal septum cutaneous island flap: a new flap in forefoot reconstruction. Plastic and Reconstructive Surgery 1997; 99: 1390-1395.

11. Cigna E, Fioramonti P, Fino P, Scuderi N. Island lateral plantar artery perforator flap for reconstruction of weight-bearing plantar areas. Foot and ankle Surgery: official journal of the European society of Foot and ankle Surgeons 2011; 17: 13-16.

12. Bhandari PS, Sobti C. Reverse flow instep island flap. Plastic and Reconstructive Surgery 1999; 103: 1986-1989.

13. Coruh A. Distally based perforator medial plantar flap: a new flap for reconstruction of plantar forefoot defects. Annals of Plastic Surgey 2004; 53: 404-408.

14. Masuoka T, Kato A. Posterior tibial vein as drainage vessel for the medial plantar flap on the lateral plantar artery. Plastic and Reconstructive Surgery 2004; 114: 1350-1351. 


\section{Case Report}

15. Fujioka M, Hayashida K, Senju C. Reconstruction of lateral forefoot using reversed medial plantar flap with free anterolateral thigh flap. The Journal of Foot and Ankle Surgery 2014; 53: 324-327.

16. Acikel C, Celikoz B, Yuksel F, Ergun O. Various applications of the medial plantar flap to cover the defects of the plantar foot, posterior heel, and ankle. Annals of Plastic Surgery 2003; 50: 498-503.

17. Duman $\mathrm{H}, \mathrm{Er} \mathrm{E}$, Isik $\mathrm{S}$, et al. Versatility of the medial plantar flap: our clinical experience. Plastic and Reconstructive Surgery 2002; 109:
1007-1012.

18. Zelken JA, Lin CH. An Algorithm for Forefoot Reconstruction with the Innervated Free Medial Plantar Flap. Annals of Plastic Surgery 2016; 76: 221-226.

19. Kim CY, Naidu S, Kim YH. Supermicrosurgery in peroneal and soleus perforator-based free flap coverage of foot defects caused by occlusive vascular diseases. Plastic and Reconstructive Surgery 2010; 126: 499-507. 